\title{
O uso de esteróides anabolizantes em esportistas recreativos
}

\author{
Artigo Original
}

\author{
Olga Kyselovicova' \\ kyselovicova@fsport.uniba.sk \\ Branislav Antala' \\ antala@fsport.uniba.sk \\ Karol Michalak ${ }^{2}$ \\ michalak@yahoo.com
}

\author{
Comenius University - Faculty of Physical Education and Sports - \\ Bratislava - Slovakia \\ ${ }^{2}$ Comenius University - Faculty of Pharmacy - Bratislava - Slovakia
}

Kyselovicova O, Antala B, Michalak K. O uso de esteróides anabolizantes em esportistas recreativos. Fit Perf J. 2008 marabr;7(2):65-8.

RESUMO: Introdução: $\bigcirc$ objetivo deste estudo é fazer uma avaliação geral sobre o uso de esteróides anabolizantes entre os desportistas recreativos e, ao mesmo tempo, analisar outras drogas usadas para aumentar rapidamente o nível de aptidão individual dos usuários. Materiais e Métodos: Foi usado um questionário para investigar o potencial de abuso de esteróides anabolizantes entre os desportistas recreativos, que praticam exercícios sem competir, treinando no nível intermediário de três a quatro vezes por semana. Foram escolhidos aleatoriamente 80 homens $(21,6 \pm 3,4$ anos) de diversas academias, aos quais foi solicitado responder um questionário anonimamente. Dos questionários enviados, $80 \%$ (64) foram devolvidos. Resultados: As descobertas mais importantes do nosso estudo foram: o uso de esteróides anabolizantes foi confirmado por $21 \%$ de todos os que responderam; todos os usuários de anabolizantes usam as drogas sem qualquer supervisão médica profissional; $36 \%$ dos usuários de anabolizantes estão decididos a usar drogas novamente, em caso de atividade física mais pesada ou fadiga; $36 \%$ dos atuais usuários de anabolizantes estão decididos a usar os anabolizantes em caso de desempenho físico; a maioria das informações sobre os efeitos dos anabolizantes é conseguida através de atletas profissionais e "distribuidores não-oficiais"; e é comum o uso de anabolizantes e drogas estimulantes sem qualquer supervisão médica. Discussão: Os resultados deste estudo servirão para alertar sobre a dinâmica expansão do abuso de anabolizantes e avaliar o conhecido uso de anabolizantes e drogas estimulantes para melhorar o desempenho físico.

Palavras-chave: Anabolizantes, Esteróides, Aptidão Física.

Endereço para correspondência:

Comenius University, Faculty of Physical Education and Sports - L.Svobodu 9 - 81469 Bratislava - Slovakia

2




\section{Using of anabolic steroids in men's recreational fitness training}

Introduction: The aim of this study was to give general overview about the use of anabolic steroids among the recreational sportsmen and at the same time to analyse other drugs used for immediate help to increase their individual fitness level. Materials and Methods: The questionnaire was used to investigate the potential abuse of anabolic steroids among the recreational sportsmen, practising non-competitive fitness training at the intermediate level 3 to 4 times per week. Randomly selected 80 men (21.6 \pm 3.4 years) from various fitness centres were asked to fulfil the questionnaires anonymously. $80 \%$ (64) of questionnaires were returned. Results: The most significant finding of our study: use of anabolic steroids has been determined by $21 \%$ of all respondents; any of anabolic users use the drugs without any professional medical supervision; $36 \%$ of present anabolic users are decided to use drugs again in case of harder physical activity or fatigue; $36 \%$ of present non-users are decided to use the anabolics in case of physical performance; majority of information on anabolic effects is reached from professional athletes and "unofficial distributor" and common use of anabolics and stimulation drugs without any medical supervision. Discussion: The results of this study should alert to solve the dynamic expansion of anabolics abuse and evaluate the well-known use of anabolics and stimulation drugs to improve physical.

Keywords: Anabolic Agents, Steroids, Physical Fitness.
El uso de esteroides anabolizantes en deportistas recreativos

Introducción: El objetivo de este estudio es hacer una evaluación general sobre el uso de esteroides anabolizantes entre los deportistas recreativos y, a la vez, analizar otras drogas usadas para aumentar rápidamente el nivel de aptitud individual de los usuarios. Materiales y Métodos: Fue usado un cuestionario para investigar lo potencial de abuso de esteroides anabolizantes entre los deportistas recreativos, que practican ejercicios sin competir, entrenando en el nivel intermediario de tres la cuatro veces a la semana. Habían sido elegidos aleatoriamente 80 hombres (21,6 $6 \pm 3,4$ años) de diversas academias, los cuales habían sido solicitados a responder un cuestionario anónimamente. 80\% (64) de los cuestionarios habían sido devueltos. Resultados: Las descubiertas más importantes de nuestro estudio, fueron: el uso de esteroides anabolizantes fue confirmado por $21 \%$ de todos los que respondieron; todos los usuarios de anabolizantes usan las drogas sin cualquier supervisión médica profesional; $36 \%$ de los usuarios de anabolizantes están decididos a usar drogas nuevamente, en caso de actividad física más pesada o fatiga; $36 \%$ de los actuales usuarios de anabolizantes están decididos a usar los anabolizantes en caso de desempeño físico; la mayoría de las informaciones sobre los efectos de los anabolizantes es conseguida a través de atletas profesionales y "distribuidores no-oficiales"; y es común el uso de anabolizantes y drogas estimulantes sin cualquier supervisión médica. Discusión: Los resultados de este estudio servirán para alertar sobre la expansión dinámica del abuso de anabolizantes y evaluar el conocido uso de anabolizantes y drogas estimulantes para mejorar el desempeño físico.

Palabras clave: Agentes Anabolizantes, Esteroides, Acondicionamiento Físico.

\section{INTRODUÇÃO}

O problema do uso de esteróides anabolizantes por atletas consagrados vem sendo discutido amplamente nos níveis médico, ético, comportamental e experimental, por pelo menos 20 anos $^{1,2,3,4}$. Teste anti-drogas, políticas rígidas e programas educacionais continuam sendo as respostas principais ao problema. Apesar disto, o abuso de drogas no esporte competitivo continua sendo penetrante. Diversas explicações foram dadas e a razão varia da melhoria da performance ao alívio do estresse e do tédio. Porém, o uso de esteróides anabolizantes em esportes recreativos e de condicionamento é praticamente novo, não apresentando quaisquer dados relevantes. Além de pouquíssimos estudos de casos, uma carência relativa de informação foi apontada no que diz respeito à experiência subjetiva das pessoas que usam esteróides anabólico-androgênico ${ }^{5,6,7}$.

\section{Objetivo do estudo}

O objetivo deste estudo foi realizar uma avaliação geral sobre o uso de esteróides anabolizantes entre os desportistas recreativos e, ao mesmo tempo, analisar outras drogas utilizadas para aumentar rapidamente o nível de condicionamento individual. Os benefícios ou os malefícios deste fenômeno são bem co- nhecidos, mas não foram discutidos especificamente para $\circ$ esporte recreativo.

Em nosso estudo também tentamos descobrir o nível de informação sobre o efeito de drogas encorajadoras em desportistas, e as fontes, quando a procedência destas é conhecida.

\section{MATERIAIS E MÉTODOS}

\section{Aprovação do estudo}

Este estudo foi aprovado pelo Comitê de Ética da Faculdade de Farmácia da Universidade Comenius - Bratislava, sob o $\mathrm{n}^{\circ}$ $659 / 2006$.

\section{Caracterização do estudo}

Foi utilizado um questionário para investigar o abuso em potencial de esteróides anabolizantes entre os desportistas recreativos, que praticavam exercícios sem competir, treinando de três a quatro vezes por semana. Foram escolhidos aleatoriamente 80 homens $(21,6 \pm 3,4$ anos) de diversas academias, aos quais foi solicitado responder um questionário anonimamente. Destes, $80 \%$ (64) foram devolvidos. 
Tabela 1 - Estado de saúde de usuários de esteróides anabolizantes

\begin{tabular}{lc}
\hline problemas de saúde & $\begin{array}{c}\text { \% de usuários de esteróides } \\
\text { anabolizantes }\end{array}$ \\
\hline dor em órgãos de movimento & 63 \\
dor de cabeça freqüente & 9 \\
fadiga constante & 9 \\
\hline
\end{tabular}

As perguntas foram divididas em quatro grupos, abordando os seguintes tópicos:

1. estado da saúde e parâmetros básicos somatométricos, periodicidade e duração das atividades esportivas realizadas;

2. presença e freqüência do uso de drogas entre os atletas;

3. informação sobre ações "positivas" esperadas e possíveis efeitos colaterais de esteróides de anabolizantes usados; e

4. avaliação de efeitos percebidos no uso de esteróides anabolizantes, pelos próprios usuários.

\section{RESULTADOS}

Como esperado, a comparação de parâmetros somatométricos mostrou que o Índice de Massa Corporal comum em usuários de esteróides anabolizantes era significativamente mais alto do que em não-usuários: $27,9 \pm 2,1 \mathrm{~kg} \cdot \mathrm{m}^{-2}$ e $22,3 \pm 2,7 \mathrm{~kg} \cdot \mathrm{m}^{-2}$, respectivamente.

A análise de estado de saúde de usuários de anabolizantes é mostrada na Tabela 1 .

\section{DISCUSSÃO}

\section{Uso de esteróides anabolizantes para os efeitos proteoanabólicos}

Os resultados (Figura 1) mostraram que $21 \%$ das pessoas envolvidas nesta investigação fizeram uso de esteróides anabolizantes, como estonasol, testosterona, derivados, superanabolônio, andriol, sustanon, nandrolon e metandrostolon. Dos sujeitos que retornaram o questionário, 18\% usaram drogas paralelamente com algum outro tipo de farmacoterápico prescrito.

Em todos os casos de esportistas recreativos, os esteróides anabolizantes foram usados visando os efeitos proteoanabolizantes sem qualquer controle médico, supervisão ou consulta. Portanto, todos os esteróides foram obtidos de fontes não-oficiais. Dos que responderam, 63\% usaram drogas por um período limitado de tempo, como um teste, e $9 \%$ usaram regularmente. Um dos fatos mais significantes é que $36 \%$ dos indivíduos estão abertos a usarem esteróides no futuro. Apenas $27 \%$ alegaram que não pretendiam usar esteróides novamente. Adicionalmente, $72 \%$ de todos que responderam confirmaram conhecimento, tanto dos efeitos positivos quanto dos não-desejados.
Figura 1 - Uso de esteróides anabolizantes para o efeito proteoanabolizante

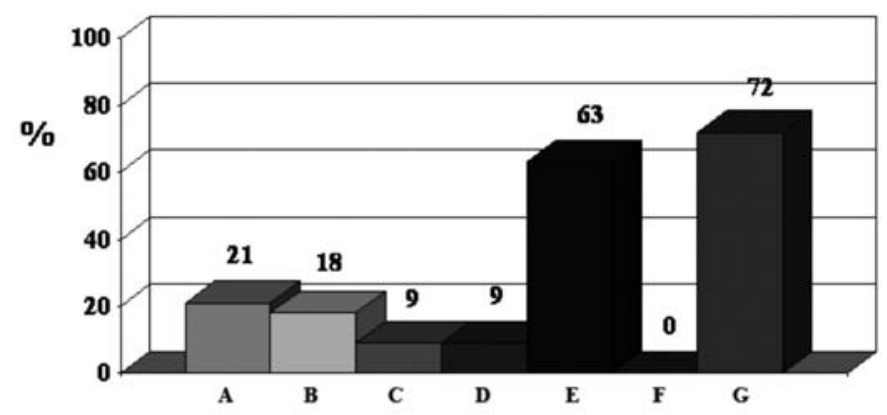

A Número de usuários de anabolizantes

B Uso concomitante com outras substâncias farmacológicas prescritas

C Uso regular em longo prazo

D Uso em caso de fadiga

E Uso para teste

F Uso sob supervisão médica

G Conhecimento sobre ações "positivas" e efeitos colaterais

Figura 2 - A informação sobre drogas e fontes de esteróides anabolizantes

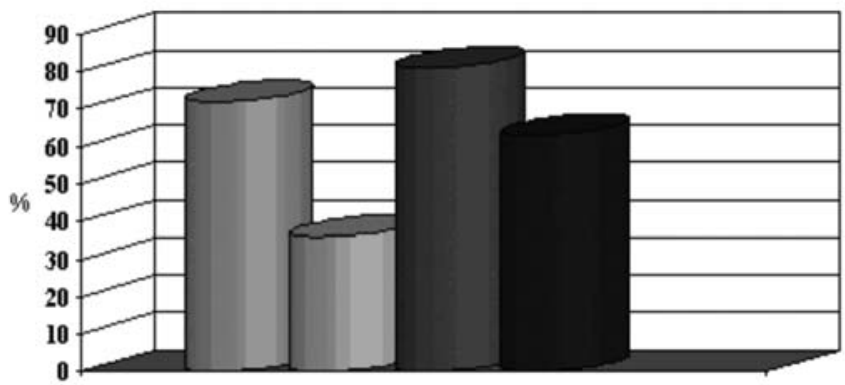

$\square$ revistas $\square$ médicos $\square$ atletas profissionais $\square$ distribuidor não-oficial

\section{Informação sobre drogas e fontes de esteróides ana- bolizantes}

Como mostrado na Figura 2, a maioria das informações veio de atletas profissionais (81\%), revistas (72\%), e de "distribuidores não-oficiais" (63\%). Nenhum dos esportistas recreativos tomou o remédio com, pelo menos, um mínimo de informação. Ao contrário, "distribuidores não-oficiais" são a fonte mais freqüente para a compra de esteróides (90\%).

\section{Efeitos colaterais esperados dos esteróides anabo- lizantes}

Os efeitos colaterais esperados mais freqüentes são o aumento de desempenho físico (100\%) e a diminuição de fadiga (81\%). Como mostrado na Figura 3, a razão para o uso de drogas é:

- redução de dor nas juntas: 54\%;

- melhora da resistência física: 27\%;

- mau humor: $27 \%$; 


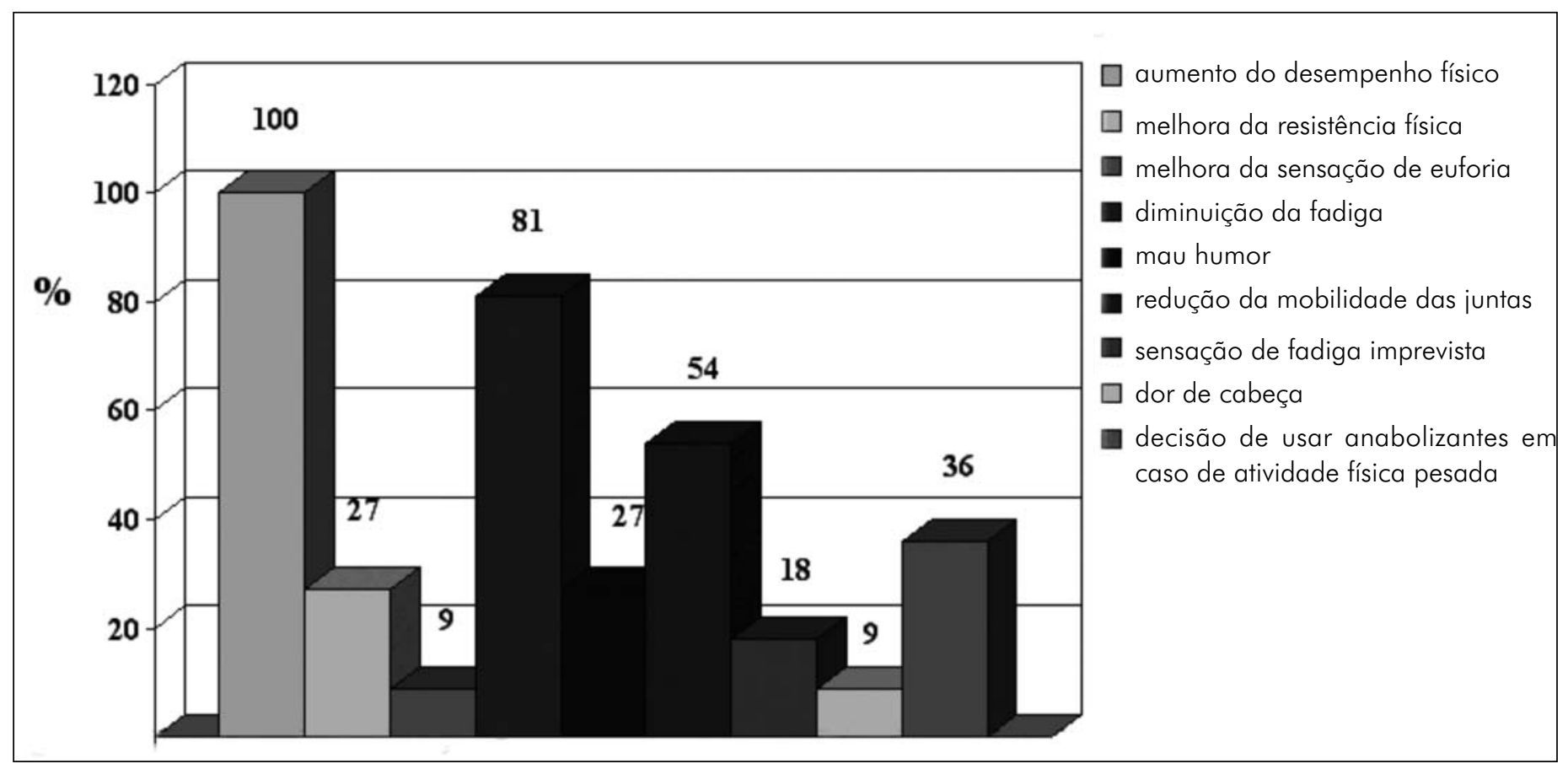

- sensação de fadiga: 18\%;

- melhora da sensação de euforia: $9 \%$; e

- diminuição de dor de cabeça: 9\%.

Apesar do conhecimento de efeitos colaterais negativos, $36 \%$ dos que responderam estão decididos a usar anabolizantes em caso de atividade física pesada.

\section{A descoberta mais significante de nosso estudo}

1. $\bigcirc$ uso de esteróides anabolizantes foi determinado por $21 \%$ de todos os que responderam. Todos os usuários de anabolizantes usam as drogas sem qualquer supervisão médica profissional.

2. $36 \%$ dos usuários de anabolizantes estão decididos a usar drogas novamente em caso de atividade física mais pesada ou fadiga.

3. $36 \%$ dos não-usuários estão decididos a usar os anabolizantes em caso de desempenho físico.

4. A maioria das informações sobre os efeitos dos anabolizantes é conseguida através de atletas profissionais e "distribuidores não-oficiais".

5. Uso comum de anabolizantes e drogas estimulantes sem qualquer supervisão médica.

Na base de nossa investigação, concluímos que o nível de informação sobre os efeitos negativos das drogas, entre esportistas recreativos e de condicionamento não-competitivo, são muito baixos. Sugerimos discutir abertamente o problema do uso de esteróides anabolizantes androgênicos nesta área.

Os resultados deste estudo deverão alertar para:

- resolver a expansão do abuso de anabolizantes; e

- avaliar o bem conhecido uso de anabolizantes e drogas estimulantes para melhorar o desempenho físico em nível não-competitivo.

\section{REFERÊNCIAS BIBLIOGRÁFICAS}

1. Luke JL, Farb A, Virmani R, Sample RH. Sudden cardiac death during exercise in a weight lifter using anabolic androgenic steroids: pathological and toxicological findings. J Forensic Sci. 1990 nov;35(6):1441-7.

2. Faigenbaum AD, Zaichkowsky LD, Gardner DE, Micheli LJ. Anabolic steroids use by male and female middle school students. Pediatrics. 1998;101(5):E6.

3. Maganaris CN, Collins D, Sharp M. Expectancy effect and strength training: do steroids make a difference? The Sport Psychologist. 2000;14(3):1224-6.

4. Woodwiss AJ, Trifunovic B, Philippides M, Norton GR. Effects of an androgenic steroid on exercise-induced cardiac remodeling in rats. J Appl Physiol. 2000 fev;88(2):409-15.

5. Fernandes Filho J. A Prática da avaliação física. Rio de Janeiro: Shape; 2003.

6. Olrich TW, Ewing ME. Life on steroids: bodybuilders describe their perceptions of the anabolic-androgenic steroid use periods. The Sport Psychologist. 1999;13(3):1131-8.

7. Corbin CB, Feyrer-Melk SA, Phelps C, Lewis L. Anabolic steroids: a study of high school athletes. Pediatr Exerc Sci. 1994 mai;6(2):149-58. 